Article

\title{
Enhancing the Cannabidiol (CBD) Compound in Formulated Hemp (Cannabis sativa L.) Leaves through the Application of Hot-Melt Extrusion
}

\author{
Md Obyedul Kalam Azad ${ }^{1,2,+}$ () Byeong Ryeol Ryu ${ }^{1,+}{ }^{-}$, Md Soyel Rana ${ }^{1}$, Md Hafizur Rahman ${ }^{1}$, \\ Jung-Dae $\operatorname{Lim}^{1}$ D and Young-Seok Lim ${ }^{1,2, *}$ \\ 1 Department of Bio-Health Convergence, Kangwon National University, Chuncheon 24341, Korea; \\ azadokalam@gmail.com (M.O.K.A.); fbqudfuf0419@naver.com (B.R.R.); ranar0ranar@gmail.com (M.S.R.); \\ hafiz120894@gmail.com (M.H.R.); ijdae@kangwon.ac.kr (J.-D.L.) \\ 2 Valley Food Tec., 2112, Highway 25, Eden, ID 83325, USA \\ * Correspondence: potatoschool@kangwon.ac.kr; Tel.: +82-33-250-6474 \\ + Both authors contributed equally.
}

Citation: Azad, M.O.K.; Ryu, B.R.; Rana, M.S.; Rahman, M.H.; Lim, J.-D.; Lim, Y.-S. Enhancing the Cannabidiol (CBD) Compound in Formulated Hemp (Cannabis sativa L.) Leaves through the Application of Hot-Melt Extrusion. Processes 2021, 9, 837. https://doi.org/10.3390/pr9050837

Academic Editor: Shu-Yao Tsai

Received: 10 April 2021

Accepted: 7 May 2021

Published: 11 May 2021

Publisher's Note: MDPI stays neutral with regard to jurisdictional claims in published maps and institutional affiliations.

Copyright: (C) 2021 by the authors. Licensee MDPI, Basel, Switzerland. This article is an open access article distributed under the terms and conditions of the Creative Commons Attribution (CC BY) license (https:/ / creativecommons.org/licenses/by/ $4.0 /)$.

\begin{abstract}
Cannabidiol (CBD) is a non-psychoactive cannabinoid compound found in hemp plants that has recently sparked interest in the biomedical and food industries. CBD is a natural decarboxylated product of cannabidiolic acid (CBDA). In this study, processing parameters were developed to enhance the decarboxylation process of CBDA in hemp leaves using hot-melt extrusion (HME). The hemp leaves were formulated with two different acid-based polymers, namely ascorbic acid (AA) and ascorbyl palmitate (AP), before the HME. The results showed that the carboxylation process of CBDA was increased by at least 2.5 times in the extrudate leaves and the content of the CBD was four times higher when formulated with AP $(2800 \mu \mathrm{g} / \mathrm{g})$ compared with the raw leaves $(736 \mu \mathrm{g} / \mathrm{g})$. The total phenolic and total flavonoid content, as well as the DPPH antioxidant capacity, were higher in the AP formulated extrudate. At the same time, the $\Delta 9$-tetrahydrocannabinol (THC) content was reduced by half in the extrudate compared with the raw leaves. It was also observed that double HME processing did not increase the decarboxylation process. It was concluded that the HME process significantly improved the conversion rate of CBDA to CBD in formulated hemp leaves with a reduced THC content.
\end{abstract}

Keywords: biopolymer; formulation; decarboxylation; bioactive compounds; antioxidant capacity

\section{Introduction}

Cannabidiol (CBD) is a non-psychoactive and non-intoxicating cannabinoid compound found primarily in hemp plants' leaves and inflorescence. It is a 21-carbon terpenophenolic compound that is produced when a cannabidiolic acid (CBDA) precursor is decarboxylated (Figure 1) [1,2]. CBD has recently gained much attention from the pharmaceuticals industry because of the versatile potential utilization of these compounds including for epilepsy, as well as for its cancer anti-inflammatory, analgesic, antioxidant, antimicrobial, neuro-protective, and anticonvulsant properties [2-5]. Cannabinoid compounds, including cannabidiol (CBD) and $\triangle 9$-tetrahydrocannabinol (THC), are derived from their precursor, cannabigerolic acid (CBGA). THC/THC acid (THCA) and CBD/CBDA are the subsequent synthesized products of CBGA. The THCA and CBDA compounds are decarboxylated to THC and CBD, respectively, when exposed to heat and light [2].

More than 100 cannabinoids have been extracted and detected in hemp plants; among them, THC is a psychoactive compound that is subject to strict legal restrictions in most countries, despite its widespread acceptance by patients and medical professionals [6]. However, the CBD compound is considered to be one of the principal non psychoactive prevalent compounds, and has been appraised and highly touted for use in many disorders that have been licensed by the US Food and Drug Administration (FDA) [7]. Studies have 
reported that the health benefits of $\mathrm{CBD}$ and $\mathrm{CBD}$ infused products have had overwhelming breakthroughs or even miracles for many diseases [8]. In the last few years, CBD containing food products have also been greatly increased in US and Europe markets [9].

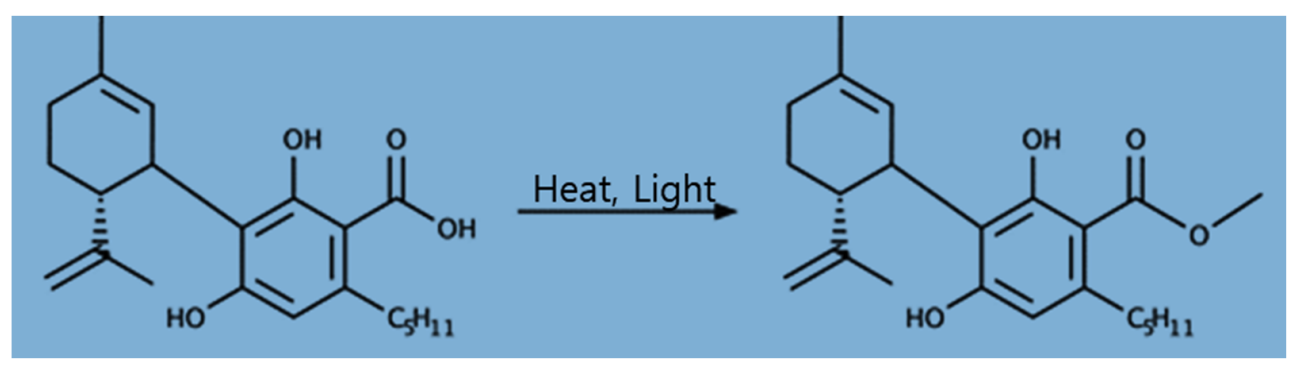

Figure 1. Chemical structure of cannabidiolic acid (CBDA; left) and cannabidiol (CBD; right).

Decarboxylation is an important step in order to obtain a higher yield of CBD. Research has shown that naturally, the amount of CBD in hemp plants is not significant, but its concentration can be increased by inducing a decarboxylation elicitor, such as heat and light [10]. The efficient production of CBD from hemp plants is crucial in order to boost the successful use of hemp plants in the medical and food industries, as CBDA is pharmacologically inactive [11]. The CBDA compound is thermally unstable and can be decarboxylated to $\mathrm{CBD}$. The decarboxylation process starts from 80 to $230{ }^{\circ} \mathrm{C}$, depending on the duration of the exposure time [12].

In this study, we attempted to optimize the decarboxylation process of CBDA so as to get a higher yield of CBD and to reduced THC content in the hemp leaves through the application of hot-melt extrusion technology (HME). One goal of HME is to design, create, and commercialize industrial processes that are both sustainable and economically viable, while minimizing the negative chemical processes' effects on human health and the environment [13].

HME processing technology has been widely used in food and pharmaceutical industries for a long time [14]. HME is a thermal processing technology that generates high shear forces to develop solid extrudates. HME facilitates in the breakdown or deformation of the molecular structure of the extruded molecules [15]. Because of the advantages of HME, we used it to decarboxylate the cannabinoid compounds to yield higher CBD content.

\section{Materials and Methods}

\subsection{Hemp Sample Preparation}

We used Cannabis sativa L. strain India, which was aeroponically grown in a greenhouse at the Kangwon National University, Korea. The study complied with relevant institutional and national guidelines and legislation, with research permission (license number 1770) from the Ministry of Food and Drug Safety (MFDS), Korea. The fully matured (120-130 days) plants were harvested, and the leaves were naturally dried (moisture content $<10 \%$ ) in glasshouse for one week. The dried leaves were ground and prepared as a powder, and then stored in an airtight bag for further use. To protect the thermal degradation of cannabinoids during HME, biopolymers such as ascorbyl palmitate and food grade ascorbic acid were used. Ascorbyl palmitate (AP; molecular formula $\mathrm{C}_{22} \mathrm{H}_{38} \mathrm{O}_{7}$ and molecular weight: $414.53 \mathrm{~g} / \mathrm{mol}$; Figure 2) was purchased from Berg+Schmidt, GmbH \& Co. KG, Hamburg, Germany. It is an ester formed from ascorbic acid and palmitic acid creating a fat-soluble form of vitamin $\mathrm{C}$, which is also used as an antioxidant food additive (number E304). Food grade ascorbic acid (molecular formula: $\mathrm{C}_{6} \mathrm{H}_{8} \mathrm{O}_{6}$ and molecular weight: $176.12 \mathrm{~g} / \mathrm{mol}$ ) was purchased from ES food.kr. 


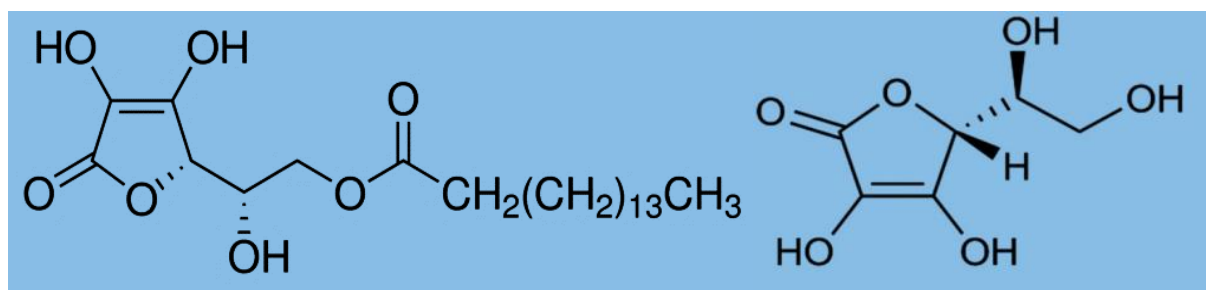

Figure 2. Chemical structure of ascorbyl palmate (left) and L ascorbic acid (right).

\subsection{Formulation Preparation}

The dried hemp leaf powder, food grade arcorbyl palmate, and L ascorbic acid were mixed according to Table 1. Formulation F0 consisted of raw fresh hemp leaf powder; F1 and F2 were prepared with raw fresh leaf powder with ascorbic acid and palmitate, respectively; F3 was an extrudate of fresh leaves; and F4 and F5 were made with first extrudate leaves (F3) with ascorbic acid and palmitate, respectively. F4 and F5 were formulated to explore the double extrusion effect on the decarboxylation process.

Table 1. Formulation ratios of the hemp leaves and biopolymers.

\begin{tabular}{ccc}
\hline Mixing Ratio & Formulation & Processing Types \\
\hline Fresh leaf $100 \%$ & F0 & No extrusion \\
F0 $80 \%+$ ascorbic acid $20 \%(w / w)$ & F1 & First extrudate \\
F0 $80 \%+$ ascorbyl palmate $20 \%(w / w)$ & F2 & First extrudate \\
Extrudate leaf $100 \%$ (without polymer) & F3 & First extrudate \\
F3 $80 \%+$ ascorbic acid $20 \%(w / w)$ & F4 & Second time extrusion of F3 \\
F3 $80 \%+$ ascorbyl palmate $20 \%(w / w)$ & F5 & Second time extrusion of F3 \\
\hline
\end{tabular}

\subsection{Development of Decarboxylated Hemp Product by HME}

The formulated hemp leaves were put into the HME feeding hopper at a feeding rate $50-100 \mathrm{~g} / \mathrm{min}$ (feeder speed 10-15 rpm), and water was added to the HME barrel at 60-70 mL/min using an auto electric pump (Festina Namsung Co., Seoul, Korea). The HME conditions were as follows: low shear, screw speed of $1000 \mathrm{rpm}$, barrel temperature of 80-90-120 ${ }^{\circ} \mathrm{C}$, and an extruder plate die of $3 \mathrm{~mm}$. The HME configuration was as follows: model FX40 Extruder (Festina FX40 Namsung Co., Seoul, Korea); main motor with 14 KW; feeder motor with $1.5 \mathrm{KW}$; production capacity of $80 \mathrm{~kg} / \mathrm{h}$; twin screw and screw speed of $1750 \mathrm{rpm}$ (max); screw diameter of $\phi 44 \times 860 \mathrm{~L}$; power supply of $200-400 \mathrm{~V} \pm 10 \% / 60 \mathrm{~Hz}$; HME sizes of W-1200, L-1900, and H-1800; and a total machine weight of 1 ton. The steps for the entire decarboxylated process are depicted in Figure 3.

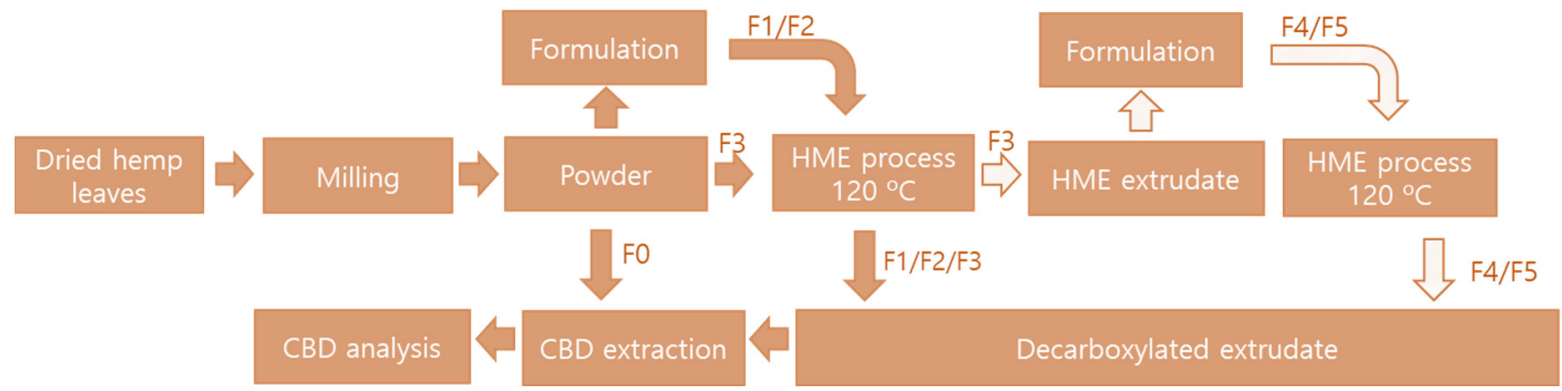

Figure 3. Schematic illustration of the entire decarboxylation processing steps.

The collected extrudate materials were dried in an oven at $50{ }^{\circ} \mathrm{C}$ for $24 \mathrm{~h}$. The dried materials were kept in an airtight bag and store at $4{ }^{\circ} \mathrm{C}$ in the refrigerator for further use. The morphology of the fresh hemp leaf powder and extrudate decarboxylated hemp leaf powder is shown in Figure 4. 


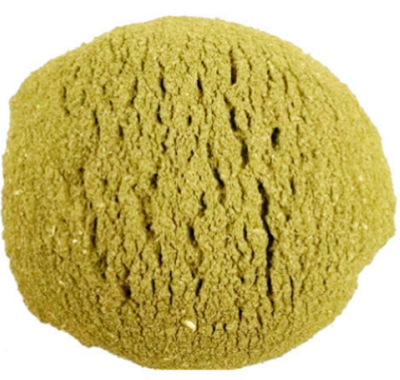

(A)

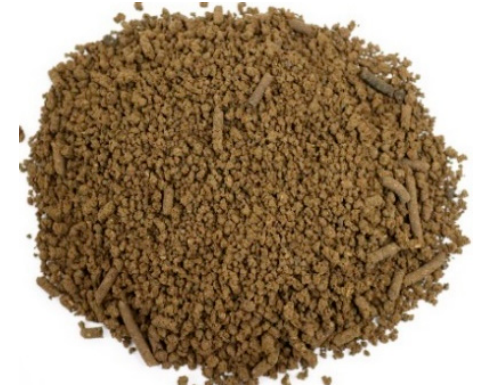

(B)

Figure 4. The (A) fresh and (B) decarboxylated extrudate hemp leaves.

\subsection{Extraction of Cannabinoid Compounds from the Raw and Decarboxylated Extrudate Materials}

About $3 \mathrm{~g}$ of dried sample was added with $27 \mathrm{~mL}$ of methanol in a conical tube. The conical tube containing the sample was sonicated using a sonicator (JEIO Tech Co., Ltd., Daejeon, Korea) for $30 \mathrm{~min}$ at room temperature. The sonicated sample was then centrifuged at $20,000 \mathrm{rpm}$ at $37^{\circ} \mathrm{C}$ for $10 \mathrm{~min}$. The supernatant was collected and evaporated through a rotatory evaporator (EYLA N-1000, Tokyo, Japan) at $40^{\circ} \mathrm{C}$. By dissolving the crude extract in $80 \%$ methanol, the stock solution $(10 \mathrm{mg} / \mathrm{mL})$ was prepared and stored at $-20^{\circ} \mathrm{C}$ for further study. All of the extraction procedures followed the standard level of the Food and Drug Administration (FDA).

\subsection{Chromatographic Analysis of Cannabinoid Compounds}

High-performance liquid chromatography (HPLC; Shimadzu LC-20AT HPLC system) was used to analyze the CBDA/CBD and THCA/THA from the fresh and decarboxylated extrudate hemp leaves, according to the method of Jin et al. [16]. The HPLC chromatographic method was validated and optimized according to FDA guidelines. The parameters that were evaluated during method development were specificity, linearity, limits of detection (LOD) and limits of quantitation (LOQ), range, accuracy, and precision [16]. All of the other parameters, such as operating parameters, reagents, standards control sample, and calculations, also maintained the FDA standard. The column used in this analysis was Reverse phase Zorbax SB-C 18 ( $4.6 \mathrm{~mm} \times 100 \mathrm{~mm}, 3.5 \mu \mathrm{m})$, with a mobile phase of $70 \%$ acetonitrile containing $0.1 \%$ formic acid in isocratic mode, with a flow rate of $1.5 \mathrm{~mL} / \mathrm{min}$ (for CBDA/THCA) and $1.0 \mathrm{~mL} / \mathrm{min}$ (for CBD/THC), injection volume of $10 \mu \mathrm{L}$, oven temperature of $30^{\circ} \mathrm{C}$, and detectors of $275 \mathrm{~nm}$ (for CBDA/THCA) and $220 \mathrm{~nm}$ (for CBD/THC). A standard calibration curve was plotted for the absorption of CBDA and CBD (Cerilliant, Round Rock, TX, USA) showing the standard and peak area of the standard sample. The contents of the cannabinoid (three replications) were expressed as $\mu \mathrm{g} / \mathrm{g}$ on the dry matter basis.

\subsection{Estimation of the Total Phenol, Flavonoid Content, and Antioxidant Capacity}

The total phenol content (TPC) was measured according to the Folin-Ciocalteau process [17]. Briefly, $200 \mu \mathrm{L}$ of Folin-Ciocalteau reagent (1N) was added to a test tube containing $1 \mathrm{~mL}$ of sample $(1 \mathrm{mg} / \mathrm{mL})$. The volume of the mixture was increased by adding deionized water $(1.8 \mathrm{~mL}$ ) and was kept ( $3 \mathrm{~min}$ at room temperature) before being vortexed. Afterward, $400 \mu \mathrm{L}$ of sodium carbonate $(10 \% v / v)$ was added to the reaction mixture. Finally, $600 \mu \mathrm{L}$ of deionized water was added to adjust the volume up to $4 \mathrm{~mL}$, and it was placed in dark ambience for $1 \mathrm{~h}$ of incubation. The absorbance was measured with a spectrophotometer (UV-1800 240 V, Shimadzu Corporation, Kyoto, Japan) against the control at $725 \mathrm{~nm}$. The TPC was calculated from a calibration curve (plotting the value of absorbance vs. concentration) using gallic acid, and was expressed as mg of GAE/100 g of sample.

According to the previously mentioned procedure, the total flavonoid content (TFC) was calculated with some modifications [18]. In brief, $0.5 \mathrm{~mL}$ of sample $(1 \mathrm{mg} / \mathrm{mL})$ was 
added to $100 \mu \mathrm{L}$ of aluminum nitrate (10\% wt/vol), $100 \mu \mathrm{L}$ of potassium acetate $(1 \mathrm{M})$, and $3.3 \mathrm{~mL}$ of distilled water. The mixture was vortexed and placed in incubation (for $40 \mathrm{~min}$ at room temperature). The sample's TFC content was measured using a spectrophotometer (UV-1800 $240 \mathrm{~V}$, Shimadzu Corporation, Kyoto, Japan) at $415 \mathrm{~nm}$ and was expressed in $\mathrm{mg} / 100 \mathrm{~g}$ CBGA equivalents.

\subsection{DPPH Free Radical-Scavenging Capacity}

The antioxidant activity was determined by scavenging DPPH (2,2-diphenyl-1 picryl hydroxyl) free radicals, following the method from the authors of [19]. In short, $3 \mathrm{~mL}$ of freshly prepared DPPH $(0.004 \% \mathrm{wt} / \mathrm{vol}$ in methanol) was added to the $1 \mathrm{~mL}$ of stock solution $(1 \mathrm{mg} / \mathrm{mL})$. The reaction mixture was vortexed and placed in the dark ambience for incubation (30 min at room temperature). The scavenging of DPPH was measured at $517 \mathrm{~nm}$ using the spectrophotometer (UV-1800 240 V, Shimadzu Corporation, Kyoto, Japan). The percentage of the scavenging capacity was calculated against the negative control (methanol + DPPH) and was expressed by the following equation:

$$
\text { Scavenging effect }(\%)=[(\text { Absc }- \text { Abss }) / \text { Absc }] \times 100
$$

where Absc is the absorbance of control and Abss is the absorbance of DPPH radical + sample (extract/standard).

\section{Statistical Analysis}

All of the data were expressed as mean \pm standard deviation (SD) of the triplicate measurements using the excel program. The obtained data of the cannabinoid content (CBD/CBD and THC/THCA) were compared with the formulations of extrudate hemp leaves using a paired t-test, so as to observe significant differences at a level of $5 \%$. Paired t-tests considering the mean values within each cannabinoid content with the different treatments were conducted using MINITAB (version 17.0, Minitab Inc., State College, PA, USA).

\section{Result and Discussions}

The extracted cannabinoids from the decarboxylated and fresh hemp leaves are shown in Table 2. It was observed that the content of CBD in the decarboxylated leaves was about three times higher compared with the raw leaves. The CBD content in the raw leaves was $736.69 \mu \mathrm{g} / \mathrm{g}$, whereas the highest CBD content was found to be $2800 \mu \mathrm{g} / \mathrm{g}$ in the F2 formulation. It was observed that without polymer mixes, the extrudate leaves (F3) achieved $1959 \mu \mathrm{g} / \mathrm{g}$ CBD, which was three times higher than for F0. It was observed that only HME had a tremendous effect on the decarboxylation process of the CBDA; however, the polymer mixed decarboxylated leaves enhanced the CBD content more than those without polymer mixed decarboxylated leaves.

Table 2. The CBD and CBDA content of fresh and decarboxylated hemp leaves.

\begin{tabular}{ccc}
\hline Formulation & CBD $(\mu \mathrm{g} / \mathrm{g})$ & CBDA $(\mu \mathrm{g} / \mathrm{g})$ \\
\hline F0 & $736.7 \pm 75^{\mathrm{d}}$ & $6023.3 \pm 543^{\mathrm{a}}$ \\
F1 & $2459.0 \pm 134^{\mathrm{b}}$ & $1052.0 \pm 152^{\mathrm{b}}$ \\
F2 & $2800.3 \pm 153^{\mathrm{a}}$ & $1280.7 \pm 183^{\mathrm{b}}$ \\
F3 & $1959.0 \pm 116^{\mathrm{c}}$ & $1008.6 \pm 164^{\mathrm{b}}$ \\
F4 & $1836.2 \pm 164^{\mathrm{c}}$ & $770.2 \pm 173^{\mathrm{c}}$ \\
F5 & $2451.0 \pm 12^{\mathrm{b}}$ & $748.3 \pm 111^{\mathrm{c}}$ \\
\hline
\end{tabular}

Values marked by different letters in each column is significantly different in the $t$ test $(p<0.05)$. Mean \pm standard error (SE; $n=3)$.

The CBD content was increased four times and decarboxylation of CBDA was increased more than $78 \%$ in F2 formulation compared with F0. However, the decarboxylation rate was higher in the F5 formulation, which was $87 \%$, and the increment of CBD was 
increased by more than three times. Among the different formulations, it was observed that double extrusion was not able to speed up the decarboxylation process.

In Table 3, the psychoactive cannabinoid (THCA/THC) is demonstrated. Interestingly, it was found that the THC and THCA compounds were drastically reduced after extrusion. The THC content was $4751 \mu \mathrm{g} / \mathrm{g}$ in the raw leaves, but it was reduced to $3142 \mu \mathrm{g} / \mathrm{g}$ in F1. Likewise, THCA was reduced to $283 \mu \mathrm{g} / \mathrm{g}$ from $4389 \mu \mathrm{g} / \mathrm{g}$ in F5 to F0, respectively.

Table 3. The $\Delta 9$-tetrahydrocannabinol (THC) and THC acid (THCA) content of fresh and decarboxylated hemp leaves.

\begin{tabular}{ccc}
\hline Formulation & THC $(\mu \mathrm{g} / \mathrm{g})$ & THCA $(\mu \mathrm{g} / \mathrm{g})$ \\
\hline F0 & $4751.2 \pm 578^{\mathrm{a}}$ & $4389.5 \pm 987^{\mathrm{a}}$ \\
F1 & $3142.7 \pm 754^{\mathrm{b}}$ & $377.8 \pm 86^{\mathrm{c}}$ \\
F2 & $2249.5 \pm 578^{\mathrm{c}}$ & $533.4 \pm 101^{\mathrm{b}}$ \\
F3 & $3219.6 \pm 976^{\mathrm{b}}$ & $601.0 \pm 121^{\mathrm{b}}$ \\
F4 & $2339.4 \pm 567^{\mathrm{c}}$ & $304.1 \pm 98^{\mathrm{c}}$ \\
F5 & $2245.9 \pm 875^{\mathrm{c}}$ & $283.7 \pm 95^{\mathrm{c}}$
\end{tabular}

Values marked by different letters in each column is significantly different in the $t$ test $(p<0.05)$. Mean \pm SE $(n=3)$.

The total flavonoid content (TFC), total phenolic content (TPC), and DPPH antioxidant capacity of the fresh and extrudate leaves were also analyzed (Table 4 and Figure 5). It can be seen that TFC and TPC were significantly increased in the F2 formulation, and the DPPH antioxidant capacity was also increased in the F2 formulation.

Table 4. The TFC and TPC content of fresh and decarboxylated hemp leaves.

\begin{tabular}{ccc}
\hline Formulation & TFC $(\mathbf{m g} / \mathbf{1 0 0} \mathbf{g})$ & TPC $(\mathbf{m g} / \mathbf{1 0 0} \mathbf{g})$ \\
\hline F0 & $4711.8 \pm 112^{\mathrm{c}}$ & $7.3 \pm 2.5^{\mathrm{b}}$ \\
F1 & $5923.8 \pm 164^{\mathrm{b}}$ & $9.6 \pm 3.2^{\mathrm{b}}$ \\
F2 & $8326.4 \pm 253^{\mathrm{a}}$ & $15.4 \pm 4.3^{\mathrm{a}}$ \\
F3 & $5654.8 \pm 274^{\mathrm{b}}$ & $7.4 \pm 1.5^{\mathrm{b}}$ \\
F4 & $5176.3 \pm 321^{\mathrm{b}}$ & $12.6 \pm 4.3^{\mathrm{b}}$ \\
F5 & $5237.9 \pm 473^{\mathrm{b}}$ & $13.5 \pm 3.5^{\mathrm{a}}$ \\
\hline
\end{tabular}

Values marked by different letters in each column is significantly different in the $t$ test $(p<0.05)$. Mean $\pm \mathrm{SE}$ $(n=3)$.

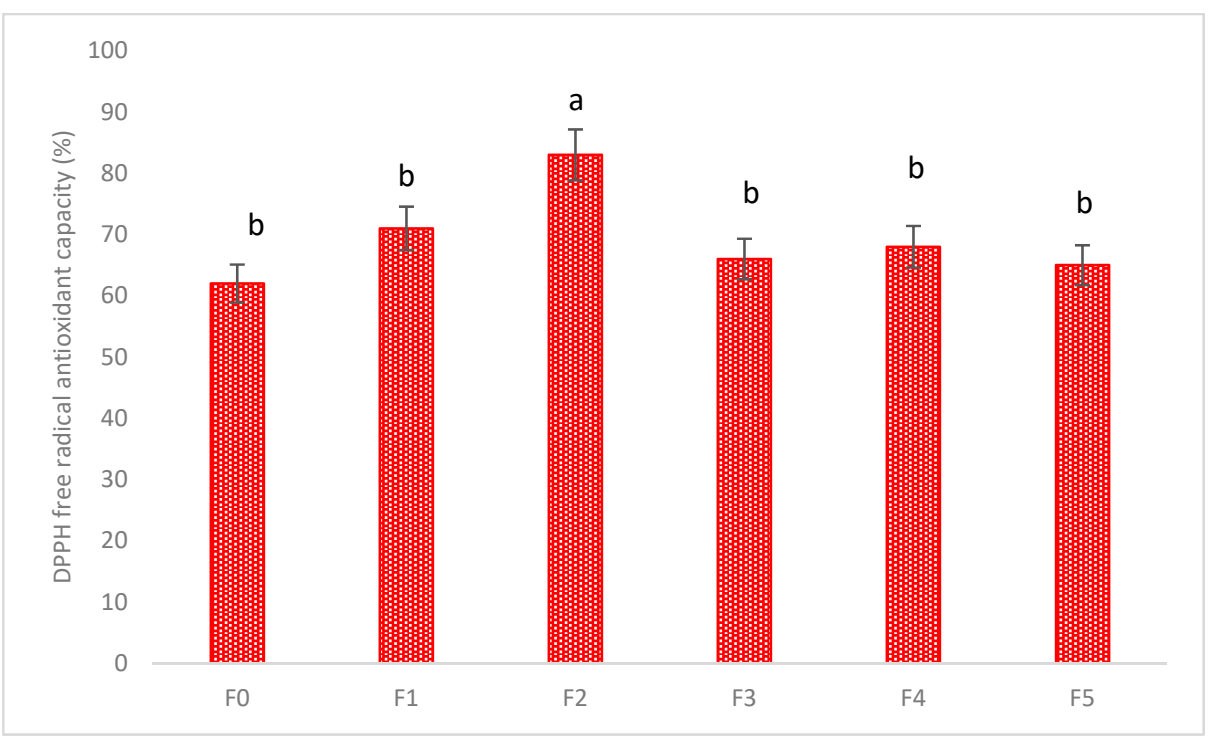

Figure 5. The DPPH antioxidant capacity of fresh and decarboxylated hemp leaves. Values marked by different letters in each column are significantly different in the $t$ test $(p<0.05)$. Mean \pm SE $(n=3)$. 
There are several parameters, including temperature, reaction time, oxygen exposure, moisture content of the materials, and chemical catalysts, that are important factors for the CBDA decarboxylation process. The biosynthesis of CBD occurs through CBDA synthase, which catalyzes the stereoselective oxidocyclization of cannabigerolic acid into CBDA [20]. It has been proven that the biosynthesis of CBDA/THCA is accelerated and decarboxylated to CBD and THC, respectively, after heating to $200-210{ }^{\circ} \mathrm{C}$ for $5 \mathrm{~min}$ [12], $120^{\circ} \mathrm{C}$ for $20 \mathrm{~min}$ [20], or $80-145^{\circ} \mathrm{C}$ for 5 to $10 \mathrm{~min}$ in a vacuum oven [10]. It has been shown that the decarboxylation of CBDA and THCA is accelerated by the heating process. The decarboxylations discussed above were carried out in dry conditions in a vacuum; however, with our HME technology, the process was done in moist conditions (20\% moisture) with a high shear HME.

We found that HME extrudate materials have a higher CBD content compared with non-extrudate materials, and the highest CBD content was achieved in the F2 extrudate. The decarboxylated process became faster when ascorbyl palmitate was added compared with ascorbic acid only. Even though the reason for the enhanced decarboxylation in the F2 formulation is still not clear, it is assumed that ascorbyl palmitate (ascorbic acid and palmitic acid) enhanced the decarboxylation process because of the acid nature, which prevented the oxidation of CBD during the HME process. The presence of $\mathrm{COOH} / \mathrm{OH}$ in ascorbyl palmitate could possibly act as a catalyst during processing, thus enhancing the CBD content. Another probable reason for the enhanced CBD in the extrudate leaves is because of the high moisture content of the extrudate during extrusion, which prevents the CBD from oxidation. This is the first study to evaluate the decarboxylation process in moist condition; however, other researchers have done so in dry environments $[10,12,20]$.

Likewise, the total phenolic compounds and total flavonoid compounds were increased in the F2 formulation, as HME has been a proven to enhance the beneficial compounds from plant materials [21,22]. Because of the strong correlation between the phenolic compounds and the antioxidant capacity [23], the DPPH antioxidant capacity was also significantly increased in the F2 formulation.

Interestingly, THCA and THC were oxidized during processing, and resulted in a very low THC in the extrudate hemp (Table 2). THC was oxidized in the processed extrudate because of the high heat sensitivity of the THC compounds. The biopolymers (either ascorbic acid or ascorbyl palmitate) had a negative impact on maintaining the THC during HME processing. The increased TFC and TPC in the extrudate F2 formulation were because of the HME extrusion effect. HME is the most convenient processing technology for developing solid formulations in the food and pharmaceutical industries [21]. The high shear-oriented screw design with a high-pressure facilitates bioactive compounds in being released from their complex molecular bonding and structure [23]. The twin screw of the HME rapture the cell wall of the molecules is thus released from the compounds [20].

\section{Conclusions}

The decarboxylation process was successfully demonstrated as an efficient CBD conversion technique using HME. It proved that CBD conversion can be enhanced when acidic biopolymer (ascorbyl palmitate) is added during HME. Therefore, it is concluded that the F2 formulation could successfully be used to obtain a higher CBD content from CBDA through the demonstrated HME process. Further investigations are needed in order to optimize the HME configurations (temperature, shear, and pressure), so as to enhance the decarboxylation process and result in zero THC.

Author Contributions: M.O.K.A. designed the study; M.O.K.A. and B.R.R. conducted, analyzed, and drafted the manuscript; M.S.R. and M.H.R. helped conduct the study and revised the manuscript; J.-D.L. and Y.-S.L. supervised the study. All authors have read and agreed to the published version of the manuscript.

Funding: This research was funded by the Chuncheon City Hemp Project, Gwangwon Do, Chuncheon, Korea. 
Institutional Review Board Statement: Not applicable.

Informed Consent Statement: Not applicable.

Data Availability Statement: Not applicable.

Acknowledgments: This research received a grant from the project "Research and Development of Chuncheon Hemp".

Conflicts of Interest: The authors declare no conflict of interest.

\section{References}

1. Andre, C.M.; Hausman, J.F.; Guerriero, G. Cannabis sativa: The plant of the thousand and one molecules. Front. Plant. Sci. 2016, 7, 1-17. [CrossRef]

2. Russo, E.B. Cannabidiol claims and misconceptions. Trends Pharmacol. Sci. 2017, 38, 198-201. [PubMed]

3. Ibeas Bih, C.; Chen, T.; Nunn, A.V.W.; Bazelot, M.; Dallas, M.; Whalley, B.J. Molecular targets of cannabidiol in neurological disorders. Neurotherapeutics 2015, 12, 699-730. [CrossRef]

4. Devinsky, O.; Marsh, E.; Friedman, D.; Thiele, E.; Laux, L.; Sullivan, J.; Miller, I.; Flamini, R.; Wilfong, A.; Filloux, F.; et al. Cannabidiol in patients with treatment-resistant epilepsy: An open-label interventional trial. Lancet Neurol. 2015, 15, 270-278. [CrossRef]

5. Ribeiro, A.; Almeida, V.I.; Costola-de-Souza, C.; Ferraz-de-Paula, V.; Pinheiro, M.L.; Vitoretti, L.B.; Gimenes-Junior, J.A.; Akamine, A.T.; Crippa, J.A.; Tavares-de-Lima, W.; et al. Cannabidiol improves lung function and inflammation in mice submitted to LPS-induced acute lung injury. Immunopharmacol. Immunotoxicol. 2015, 37, 35-41. [CrossRef]

6. $\quad$ Satterlund, T.D.; Lee, J.P.; Moore, R.S. Stigma among California's medical marijuana patients. J. Psychoact. Drugs 2015, $47,10-17$.

7. Atakan, Z. Cannabis, a complex plant: Different compounds and different effects on individuals. Ther. Adv. Psychopharmacol. 2012, 2, 241-254. [CrossRef] [PubMed]

8. Pratt, M.; Stevens, A.; Thuku, M.; Butler, C.; Skidmore, B.; Wieland, L.S.; Clemons, M.; Kanji, S.; Hutton, B. Benefits and harms of medical cannabis: A scoping review of systematic reviews. Syst. Rev. 2019, 8, 1-35. [CrossRef]

9. Walker, L.A.; Koturbash, I.; Kingston, R.; ElSohly, M.A.; Yates, C.R.; Gurley, B.J.; Khan, I. Cannabidiol (CBD) in dietary supplements: Perspectives on science, safety, and potential regulatory approaches. J. Diet. Suppl. 2020, 16, 1-10.

10. Wang, M.; Wang, Y.H.; Avula, B.; Radwan, M.M.; Wanas, A.S.; van Antwerp, J.; Parcher, J.F.; ElSohly, M.A.; Khan, I.A. Decarboxylation study of acidic cannabinoids: A novel approach using ultra-high-performance supercritical fluid chromatography/photodiode array-mass spectrometry. Cannabis Cannabinoid Res. 2016, 1, 262-271.

11. Grotenhermen, F. Pharmacokinetics and pharmacodynamics of cannabinoids. Clin. Pharm. 2003, 42, 327-360. [CrossRef]

12. Eichler, M.; Spinedi, L.; Unfer-Grauwiler, S.; Bodmer, M.; Surber, C.; Luedi, M.; Drewe, J. Heat exposure of Cannabis sativa extracts affects the pharmacokinetic and metabolic profile in healthy male subjects. Planta Med. 2012, 78, 686-691. [CrossRef]

13. Alshetaili, A.; Alshahrani, S.M.; Almutairy, B.K.; Repka, M.A. Hot Melt Extrusion Processing Parameters Optimization. Processes 2020, 8, 1516. [CrossRef]

14. Repka, M.A.; Majumdar, S.; Battu, S.K.; Srirangam, R.; Upadhye, S.B. Applications of hot-melt extrusion for drug delivery. Expert Opin. Drug Deliv. 2008, 5, 1357-1376. [PubMed]

15. Jin, D.; Dai, K.; Xie, Z.; Chen, J. Secondary metabolites profiled in cannabis inflorescences, leaves, stem barks, and roots for medicinal purposes. Sci. Rep. 2020, 10, 1-14.

16. Singleton, V.L.; Rossi, J.A. Colorimetry of total phenolics with phosphomolybdic-phosphotungstic acid reagents. Am. J. Enol. Vitic. 1965, 16, 144-458.

17. Azad, M.O.K.; Kim, W.W.; Jin, C.W.; Kang, W.S.; Park, C.H.; Cho, D.H. Development of a Polymer-Mediated Soybean Nanocomposite by Hot Melt Extrusion to Improve Its Functionality and Antioxidant Properties. Foods 2019, 8, 41. [CrossRef]

18. Braca, A.; Fico, G.; Morelli, I.; Simone, F.; Tome, F.; Tommasi, N. Antioxidant and free radical scavenging activity of flavonol glycosides from different Aconitum species. J. Ethnopharmacol. 2003, 8, 63-67. [CrossRef]

19. Taura, F.; Morimoto, S.; Shoyama, Y. Purification and characterization of cannabidiolic-acid synthase from Cannabis sativa L.: Biochemical analysis of a novel enzyme that catalyzes the oxidocyclization of cannabigerolic acid to cannabidiolic acid. J. Biol. Chem. 1996, 271, 17411-17416.

20. Maniruzzaman, M.; Rana, M.M.; Boateng, J.S.; Douroumis, D. Dissolution enhancement of poorly water-soluble APIs processed by hot-melt extrusion using hydrophilic polymers. Drug Dev. Ind. Pharm. 2012, 392, 218-227. [CrossRef]

21. Azad, M.O.K.; Adnan, M.; Sung, I.J.; Lim, J.D.; Baek, J.S.; Lim, Y.S.; Park, C.H. Development of value-added functional food by fusion of colored potato and buckwheat flour through hot-melt extrusion. J. Food Process Preserv. 2021, 2021, e15312.

22. Azad, M.O.K.; Piao, J.P.; Park, C.H.; Cho, D.H. Far infrared irradiation enhances nutraceutical compounds and antioxidant properties in Angelica gigas Nakai powder. Antioxidants 2018, 7, 189. [CrossRef] [PubMed]

23. Censi, R.; Gigliobianco, M.R.; Casadidio, C.; Di Martino, P. Hot Melt Extrusion: Highlighting physicochemical factors to be investigated while designing and optimizing a hot melt extrusion process. Pharmaceutics 2018, 10, 89. [CrossRef] [PubMed] 